\title{
Diagnosing asthma in adults in primary care: a qualitative study of Australian GPs' experiences
}

\author{
*Sarah M Dennis'a, Nicholas A Zwarb, Guy B Marks ${ }^{c, d}$ \\ a Senior Research Fellow, Centre for Primary Health Care and Equity, School of Public Health and Community Medicine, University of \\ New South Wales, Sydney, Australia \\ ${ }^{\mathrm{b}}$ Professor of General Practice, School of Public Health and Community Medicine, University of New South Wales \\ c Research Leader, Epidemiology Group, Woolcock Institute of Medical Research, University of Sydney \\ ${ }^{\mathrm{d}}$ Consultant Physician, Department of Respiratory Medicine, Liverpool Hospital, Sydney
}

Originally submitted 18th October 2008; resubmitted 20th March 2009; revised version received 13th May 2009; accepted 20th May 2009; online 21st July 2009

\begin{abstract}
Aim: To explore what difficulties are faced by general practitioners (GPs) when trying to make a diagnosis of asthma in adults, and whether there are patient characteristics that influence this process.

Methods: A qualitative study in which three focus group discussions were conducted with $18 \mathrm{GPs}$.

Results: GPs were confident with the components needed to confirm a diagnosis of asthma but not with the use of spirometry. GPs said that time was an important factor - time to undertake the tests in their practice, and time to persuade the patient to engage in the diagnostic process including follow-up visits. Patients who were less willing to engage in this process were likely to be treated symptomatically.

Conclusion: Continuity of care is important. A diagnosis of asthma seems to be more likely in patients with an ongoing relationship with their GP where both are willing to engage in the diagnostic process.

(C) 2010 Primary Care Respiratory Society UK. All rights reserved.

SM Dennis, et al. Prim Care Resp J 2010; 19(1): 52-56.

doi:10.4104/pcrj.2009.00046
\end{abstract}

Keywords asthma, diagnosis, primary health care, focus group

The full version of this paper, with online Appendix,

is available at www.thepcri.org

\section{Introduction}

Inaccurate diagnosis of asthma is a common problem in primary care worldwide. ${ }^{1-3}$ Treatment for asthma is effective in improving quality of life and ameliorating most of the adverse health outcomes of the disease. ${ }^{4}$ There is evidence that patients are more likely to receive appropriate anti-asthma medication if they have a diagnosis of asthma recorded in their medical notes., ${ }^{5,6}$

Though published guidelines outline the criteria necessary for clinical diagnosis, making a diagnosis of asthma can be difficult and there is often uncertainty.-9 GPs are encouraged to use spirometry or peak expiratory flow (PEF) readings to confirm the diagnosis but this may not always be carried out. A case notes review of patients with newly identified asthma in primary care in the UK found that less than $20 \%$ of patients had the results of any airflow test recorded in their medical notes. ${ }^{10}$ Even when spirometry is undertaken the fluctuating nature of asthma means that normal spirometry at the time of the visit does not exclude a diagnosis of asthma. In an attempt to improve the diagnosis of asthma in primary care, two groups have tried to develop a formula based on symptoms. ${ }^{11,12}$ Both groups identified current wheeze and dyspnoea as important symptoms in the diagnosis of asthma; however, these are also symptoms of chronic obstructive pulmonary disease (COPD) and so may not help GPs to differentiate asthma from other respiratory conditions.

Epidemiological studies have highlighted inaccuracies in

\footnotetext{
* Corresponding author: Dr Sarah Dennis, Senior Research Fellow, UNSW Centre for Primary Health Care and Equity, GP Unit, Fairfield Hospital PO Box 5, Wetherill Park, NSW 1860, Sydney, Australia. Tel: +61296168520 Fax: +61296168400 E-mail: s.dennis@unsw.edu.au
} 
the diagnosis of asthma. A survey conducted in Sydney in the early 1990s found that only $60 \%$ of children identified as having symptoms that might be attributable to asthma had ever been given a label of asthma and only $20 \%$ were receiving treatment. ${ }^{13}$ Another survey in western Sydney found overdiagnosis of asthma, particularly in very young children. ${ }^{14}$ In a Swedish study 86 patients on an asthma register at two general practices underwent a comprehensive diagnosis evaluation for asthma; ${ }^{3}$ the diagnosis of asthma could not be substantiated in $34 \%$ of cases, and an incorrect diagnosis was recorded for $36 \%$ of the women compared to $29 \%$ of the men. Another Swedish study identified people who had been given a diagnosis of asthma in primary care and referred them to a respiratory medicine unit for comprehensive diagnostic evaluation; ${ }^{2}$ in 16 of 68 (24\%) patients who underwent this evaluation, the diagnosis was not confirmed. In the Netherlands a sample of the population was screened for respiratory symptoms and underwent spirometry; ${ }^{1}$ however, of 86 who had symptoms suggestive of asthma and evidence of airflow obstruction, only 29 (34\%) had presented to their GP about their respiratory symptoms of these, 23 (79\% of those presenting to their GP and $27 \%$ overall) were given a diagnosis of asthma in spite of evidence of obstructive airflow limitation. Furthermore, there were clear differences in the labelling of children with current symptoms of asthma in a study in the German Dutch border area; the Dutch children tended to be labelled as asthmatic and the German children tended to be labelled as having bronchitis. ${ }^{15}$ Although there may be some reservations about the criteria used to define the diagnosis of asthma in these studies, it is clear that there is substantial variation in the use of this diagnostic label in many different settings.

The reasons underlying the variation in the use of the diagnostic label of asthma are not well understood. None of the studies cited above have attempted to ascertain why GPs give some people a diagnosis of asthma and not others, even if the patients appear, on subsequent review, to have presented with similar symptoms. It is possible that some patients have characteristics that are more likely to result in a diagnosis of asthma or are more likely to visit their GP to discuss their respiratory symptoms.

The aim of this study therefore was to understand what difficulties GPs face when trying to make a diagnosis of asthma in adults, and in addition, to explore whether there are patient characteristics that affect the GP's decision to make a diagnosis of asthma.

\section{Methods}

We used a qualitative focus group design for the study. GPs in South West Sydney were recruited though purposeful sampling of a database of research-interested GPs or were identified by local Divisions of General Practice from their membership database. A letter outlining the purpose of the study was sent and those who expressed an interest in taking part were invited to attend one of the focus groups.

The three focus groups were conducted between September 2006 and February 2007. Each focus group lasted for approximately one hour with two facilitators (SD a senior research fellow, NZ a professor of general practice), one of whom collected field notes (SD) and audiotaped the session. A topic guide was prepared which highlighted the current evidence for making the diagnosis of asthma and included questions and prompts to guide the discussion (see Appendix 1 at www.thepcrj.org). The audiotapes were transcribed verbatim and the field notes used to check the validity of the transcripts. The transcripts were thematically coded and analysed using NVivo version 7, QSR International. The framework for the coding and analysis included terms to describe the process of making a diagnosis of asthma, areas of certainty or uncertainty, and the patient characteristics that might influence this process. After each focus group there was a de-brief session (SD, NZ) where the key themes emerging were discussed, followed by further discussion with GM. Focus groups were conducted until, in the view of two investigators (SD, NZ), saturation of themes had been reached. All the participants gave their written informed consent to take part in the study. The study was approved by the University of New South Wales Human Research Ethics Committee (Approval number 06069).

\section{Results}

A total of 18 GPs took part in the three focus groups; 13 were male and 5 were female. They were an ethnically diverse group of GPs, most of whom (15/18) had 10 years' or more experience as a GP.

The terms included to describe the process of making a diagnosis of asthma, the areas of certainty or uncertainty, and the patient characteristics that might influence this process, were as follows:

\section{Previous medical history}

All the GPs attending the focus groups were familiar with the Australian guidelines for asthma management ${ }^{7}$ and the components necessary to make a diagnosis of asthma. They were confident about the symptoms and signs of asthma but were less confident when using spirometry to confirm the diagnosis, particularly in small children or older people. The GPs were more confident in making a diagnosis of asthma in someone with a history of hay fever or allergies than someone with no past history of this kind. For older patients and those who were smokers, they noted the importance of ruling out other possible conditions such as lung cancer, and the need to differentiate from COPD: 
"... if they are very old patients, no past history of asthma, they come to you with symptoms of wheezing; I think I would be very careful to put asthma as my first provisional diagnosis." (Male 4, Focus Group 1)

\section{Time factors}

The GPs raised issues such as continuity of care, the availability of consultation time and the willingness of the patient to engage in the diagnostic process as impacting on the likelihood of making a diagnosis of asthma. Time was a recurrent theme and the GPs spoke of time in different ways. They talked about the time needed by the GP in order to perform tests such as spirometry:

"I am not very conscientious with the use of spirometry and often when it's the time to do it, the waiting room is too full, so I go by history and a therapeutic trial sometimes." (Female 1, Focus Group 3)

The diagnosis of asthma usually needs to be confirmed over several visits and therefore the GP has to make a decision about how likely the patient is to return for follow-up. Often this will involve a discussion about the possible diagnosis and the steps involved to gauge the likelihood that patient will return:

"Raising a possibility of asthma raises a whole series [of questions] and involves a whole conversation that you sometimes do not have time to do; and I think consciously or otherwise, you sometimes avoid that, particularly if you sense the patients are really not that interested and are not embracing that diagnosis." (Male 3, Focus Group 1)

If the GP senses that that the patient is not interested they may treat the symptoms and leave the diagnosis for another day:

"...but if they come in and they are not really wanting to engage, it makes you feel like you are not going to engage, so you are less likely to enter a decision making process; you are more likely to say, 'Well, let's not make the diagnosis today, lets just treat you, or lets just not even talk about it'." (Female 1, Focus Group 1)

"If this patient is a regular ongoing patient, then the doctor tends to spend more time with this patient. If you think it is only one person, just for a weekend, I would get the script and go." (Male 4, Focus Group 1)

\section{Recurrent episodes}

Related to time factors is the persistence and recurrence of asthma symptoms and the importance of making the connection over time. The importance of the doctor-patient relationship and continuity of care was highlighted because the GPs were more likely to make the connection over time if they were the patient's regular GP:

"Usually people come with a cough that has been there for 2 or 3 weeks. It is not like something that started today. They have been to other doctors and have been given antibiotics, given everything and it is still hanging on. I think that is when you start to think of asthma - it is something that does not want to go away, it is lingering." (Female 1, Focus Group 2)

It can also take a considerable amount of time and repeated visits to work through the diagnosis and acceptance with the patient:

"There is resistance in accepting a diagnosis - education does help, but it all takes time; it might take 2 or 3 years before it sinks in, or 2 or 3 admissions to hospital seeing the Paediatrician, or someone else in hospital to educate the patient and then it sinks in - but it can take that much longer. There is resistance to a diagnosis of asthma." (Male 3, Focus Group 2)

Persuading people to return for follow-up to confirm the diagnosis is difficult and many patients only return when they have another acute episode:

"It is very hard to get them to come back. Even with the patients that you know, you see them, you do the script, you tell them to come back on the day you are feeling better [and] we will have to do something about the matter of diagnosis, but the next time they come back is when they need another script." (Male 1, Focus Group 1)

The consequence of this is that GPs treat these patients symptomatically. When they eventually try to confirm the diagnosis of asthma they are doing so in someone who has been taking anti-asthma medication for some time:

"...the person already knows what medication they are on, they have a particular regime, they have a particular idea of their disease, because they haven't been labelled, or noone has really taken the time to diagnose them; so you are actually trying to diagnose someone that has this whole management plan underway already when they come into you for a script..... Normally you are diagnosing something from the beginning." (Female 1, Focus Group 1)

\section{Patient charactersitics}

The GPs said they were more likely to make a diagnosis of asthma in those patients who had time to hear what they were saying, and who were willing to undergo further tests and attend for follow-up. The people who were less likely to have time for the diagnostic process are professionals who are too busy to consider a diagnosis of asthma:

"It's actually the characteristics of a patient and other pressures on their life, like family or whatever it might be, that might influence the process of diagnosis as well. The process of diagnosis is to some extent patient-driven. If the patient wants to know the answer, then we tend to pursue it, but if you don't help me, we have lots of other things to do; then we are more likely to let that one go through to the keeper." (Male 5, Focus Group 1)

"....so / guess it is anxious, middle-aged women who are looking for answers in general and I think boys in their 20s do 
not really have time to hear you." (Female 1, Focus Group 1)

In addition to this, GPs felt that people with a number of chronic diseases did not take asthma as seriously as their other chronic diseases, or that asthma may be missed in favour of things higher up the list of conditions:

"Like [with] diabetes, hypertension, I found my patients are very compliant and I think the compliance with most other chronic diseases is much higher and people come for repeat prescriptions and follow up of their blood pressure, or blood sugars; but with asthma, I found it is very, very difficult." (Male 2, Focus Group 1)

\section{Awareness}

The willingness of patients to discuss the possibility of asthma and bring it up with the GP is an important factor. The GPs thought that very overweight people or smokers might not be willing to mention that they were experiencing wheeze or shortness of breath in case this led to a lecture on quitting, weight loss or exercise:

"...it is like someone who is overweight, someone who is morbidly obese or whatever, [they] might not mention it because they know that you are going to say; 'Well, what about losing some weight, or doing some exercise, or stopping smoking?'" (Female 1, Focus Group 3)

GPs reported feeling more confident making a diagnosis in someone who was younger and fitter:

"I suspect I might think of asthma sooner in a nonsmoking relatively young man, than an unfit sedentary smoker." (Male 3, Focus Group 1)

\section{Discussion}

\section{Summary of the findings}

The GPs who attended the focus groups expressed confidence about the theoretical steps involved in making a diagnosis of asthma in adults but said that in practice it was not always so straightforward. Continuity of care and the patient-GP relationship are important determinants of whether the GP is likely to invest the time and effort necessary to make a diagnosis. If the patient is unlikely to return for follow-up to confirm the diagnosis, the GPs in this study would be more likely to treat the presenting symptoms without necessarily applying a diagnostic label.

\section{Interpretation}

In Australia, general practice operates as a fee-for-service system, funded by the national medical insurance agency (Medicare). There is no capitation system so patients are not required to register with a particular GP or general practice. This has an impact on continuity of care because patients may visit different GPs or practices. If a patient visits another GP in the same practice then the doctor will have access to their medical notes but not if they visit a different practice. This will affect the GP's ability to identify repeated visits with respiratory symptoms which may be a clue to the diagnosis of asthma. If the patient is thought to be seeing more than one GP or practice and may not return to the same practice for follow-up, the GPs in this study would treat the patient symptomatically rather than entering into the process of making a diagnosis. The patient may have to pay a fee at the point of care if the GP's consultation fee is greater than the amount reimbursed by Medicare - which may be a further barrier to follow-up. The findings reported here about the problem of failure to re-attend for follow-up are consistent with findings from an evaluation of the Australian Asthma 3+ management program where patients often failed to complete the third follow-up visit with their GP within the specified time period. ${ }^{16}$

The GPs attending these focus groups were not confident about the use of spirometry and few had a practice nurse to help them with this task. The role of the practice nurse in Australia is less developed than in other countries ${ }^{17}$ and many have not undertaken post-registration training in respiratory care and may not be confident with spirometry. This, coupled with the Medicare rebate system, means that practice nurses in Australia do not usually take on spirometry and other aspects of asthma diagnosis as they do in well-organised practices in the UK. ${ }^{18}$

The patient characteristics that influence the likelihood of receiving a diagnosis of asthma may be related to their willingness to know. There are likely to be some patients with signs and symptoms of asthma who do not mention it to their GP for a variety of reasons. This is consistent with findings from a study in the Netherlands where two thirds of people with objective airflow obstruction had not discussed it with their GP. ${ }^{1}$ Instead of thinking about patient characteristics such as ethnicity or gender it may be more useful to think about how these characteristics influence the way in which patients use general practice services and their willingness to know if they have asthma or not. For example, in a Swedish study more women had an incorrect diagnosis than men. ${ }^{3}$ For the women, asthma may not have been high on their list of priorities when they saw the GP, or perhaps they had small children with them making the consultation difficult. Several studies have reported that many people take regular antiasthma medication without the diagnosis being confirmed in their medical notes, 1,10,13 and this may be because they did not have time that day or were unwilling to attend for follow-up to confirm the diagnosis so were treated symptomatically by the GP. This study highlights the fact that asthma may not be taken as seriously as other chronic diseases such as diabetes and this will have increasing significance as more people suffer from more than one chronic disease.

\section{Limitations}

A limitation of this study was that the GPs involved mostly 
worked in South West Sydney which has an ethnically diverse population and low socioeconomic status. Some of the issues raised by the GPs are likely to be exacerbated by the way in which primary care is organised in Australia and this may be less relevant to countries with a capitation system or systems where care is provided free at the point of delivery. However, many of the issues described by these GPs will still be relevant to other areas.

\section{Conclusion}

This study highlights some important issues faced by GPs when trying to establish a diagnosis of asthma in primary care. It also offers some explanation as to why some people may have a diagnosis of asthma recorded and others are taking regular treatment without a diagnosis. Continuity of care is important in primary care; ${ }^{19}$ it has been shown to improve patient care and satisfaction, and to reduce hospital admission. ${ }^{20,21} \mathrm{~A}$ diagnosis of asthma seems to be more likely in patients who have an ongoing relationship with their GP, as has been shown to be the case for diabetes. ${ }^{22}$ It will be important to maintain the public's awareness of asthma as a possible cause for their symptoms and the potential consequences of poorly controlled asthma in order to reduce complacency, particularly when other co-morbidities compete for time during GP visits.

\section{Conflict of Interest declaration}

No competing interests.

\section{Funding}

This study was funded by a Faculty Grant from the Faculty of Medicine, University of New South Wales.

\section{Acknowledgments}

The authors would like to thank all the GPs who took part in this study, and Bankstown Division of General Practice for their help in recruiting GPs and providing a venue for two of the focus groups.

\section{References}

1. van Schayck C, van der Heijden $F$, van den Boon G, Tirimanna P, van Herwaarden C. Underdiagnosis of asthma: is the doctor or patient to blame? The DIMCA project. Thorax 2000;55:562-5. http://dx.doi.org/ 10.1136/thorax.55.7.562

2. Montnemery P, Hansson L, Lanke J, et al. Accuracy of a first diagnosis of asthma in primary health care. Fam Pract 2002;19(4):365-8. http://dx.doi.org/10.1093/fampra/19.4.365

3. Marklund B, Tunsater A, Bengtsson C. How often is the diagnosis bronchial asthma correct? Fam Pract 1999;16(2):112-16. http://dx.doi.org/10.1093/fampra/16.2.112

4. Adams N, Bestall JM, Jones PW. Beclomethasone versus budesonide for chronic asthma. Cochrane Database of Systematic Reviews 2000, Issue 1. Art. No.:
CD003530. DOI: 10.1002/14651858.CD003530

5. Levy M, Bell L. General practice audit of asthma in childhood. BMJ 1984;289:1115-16. http://dx.doi.org/10.1136/bmj.289.6452.1115

6. Anderson H, Bailey P, Cooper J, Palmer J, West S. Influence of morbidity illness label and social, family and health service factors on drug treatment of childhood asthma. Lancet 1981;2:1030-32. http://dx.doi.org/10.1016/501406736(81)91225-3

7. National Asthma Council Australia. Asthma Management Handbook 2006 Melbourne; 2006 Contract No.: Document Number|.

8. British Thoracic Society, Scottish Intercollegiate Guidelines Network. British Guideline on the Management of Asthma: A national clinical guideline. Thorax 2008;63(suppl 4):iv1-iv121.

9. Global Strategy for Asthma Management and Prevention, Global Initiative for Asthma (GINA). [December 2007; cited 200811 July]; Available from: http://www.ginasthma.org

10. Dennis S, Price J, Vickers M, Frost $C$, Levy M, Barnes P. The management of newly identified asthma in primary care in England. Prim Care Resp $J$ 2002;11(4):120-2.

11. Thiadens $H$, de Bock $G$, Dekker $F$, et al. Identifying asthma and chronic obstructive pulmonary disease in patients with persistent cough presenting to general practitioners: descriptive study. BMJ 1998;316:1286-90.

12. Sistek $D$, Tschopp J-M, Schindler C, Brutsche M, Ackermann-Liebrich $U$, and the SAPALDIA team. Clinical diagnosis of current asthma: predictive value of respiratory symptoms in the SAPALDIA study. Eur Respir J 2001;17:214-19.

13. Bauman A, Young L, Peat JK, Hunt J, Larkin P. Asthma under-recognition and under-treatment in an Australian community. Aus \& NZ J Med 1992;22(1):3640.

14. Cagney $M$, Maclntyre $C$, Mclntyre P, Peat J. Childhood asthma diagnosis and use of asthma medication. Aus Fam Phys 2005;34(3):193-6.

15. Mommers M, Swaen G, Weishoff-Houben M, Dott W, van Schayck C. Differences in asthma diagnosis and medication use in children living in Germany and the Netherlands. Prim Care Resp J 2005;14:31-7. http://dx.doi.org/10.1016/j.pcrj.2004.03.008

16. Zwar NA, Comino EJ, Hasan I, Harris MF, Primary Health Care Research N. General practitioner views on barriers and facilitators to implementation of the Asthma 3+ Visit Plan. Med J Aus 2005;183(2):64-7.

17. Watts I, Foley E, Hutchinson R, Pascoe T, Whitecross L, Snowdon T. General Practice Nursing in Australia: Royal Australian College of General Practitioners, Royal College of Nursing Australia; 2004 May 2004

18. Wiener-Ogilvie S, Huby G, Pinnock H, Gillies J, Sheikh A. Practice organisational characteristics can impact on compliance with the BTS/SIGN asthma guideline: Qualitative comparative case study in primary care. BMC Family Practice 2008;9(1):32. http://dx.doi.org/10.1186/1471-2296-9-32

19. Freeman GK, Olesen F, Hjortdahl P. Continuity of care: an essential element of modern general practice? Fam Pract 2003;20(6):623-7. http://dx.doi.org/10.1093/fampra/cmg601

20. Saultz JW, Albedaiwi W. Interpersonal Continuity of Care and Patient Satisfaction: A Critical Review. Ann Fam Med 2004;2(5):445-51. http://dx.doi.org/10.1370/afm.91

21. Saultz JW, Lochner J. Interpersonal Continuity of Care and Care Outcomes: A Critical Review. Ann Fam Med 2005;3(2):159-66. http://dx.doi.org/10.1370/afm.285

22. Drivsholm T, Olivarius NdF. General practitioners may diagnose type 2 diabetes mellitus at an early disease stage in patients they know well. Fam Pract 2006;23(2):192-7. http://dx.doi.org/10.1093/fampra/cmi123

\section{Available online at http://www.thepcrj.org}


SM Dennis et al.

\section{Appendix 1}

Asthma Diagnosis Focus Group Outline

The purpose of this focus group is to discuss some of the challenges faced by GPs when making a diagnosis of asthma to try to identify some common issues faced by GPs in SW Sydney.

Because there is no 'gold standard' test and asthma is characterised by fluctuating signs and symptoms there is evidence of both under and over diagnosis of asthma in the population. Evidence suggests that patients receive the most appropriate treatment if they have an accurate diagnosis.

The National Asthma Council set out their recommendations for making a diagnosis of asthma. As there is no gold standard diagnostic test for asthma the following are recommendations for doctors:

- History

- Physical examination

- Spirometry

The purpose of this focus group is to discuss some of the challenges faced by GPs when making a diagnosis of asthma to try to identify some common issues faced by GPS in SW Sydney.

\section{Housekeeping}

- $\quad$ Sign the CPD register

- Sign the consent form if you have not already sent one

- Expenses claim to Sarah otherwise take a letter from Sarah

- CPD certificate

1. Perhaps we could start by discussing some of the challenges you face when trying to make a diagnosis of asthma?

Now let us concentrate on adults.

2. If we now reflect on asthma in adults. What aspects of history, examination and investigations are most important in making or excluding a diagnosis of asthma?

Prompt list in case they are not raised:

- Symptoms - current and pattern

- Trigger factors

- Hospital admissions

- Exacerbation profile

- Home and work environment
- Impact on work and lifestyle

- Family history of atopy

- Response to previous treatment

- $\mathrm{FEV}_{1}, \mathrm{FV}$,, $\mathrm{PEF}$ and interpretation

- Reversibility / exercise testing

- Wheezing on examination

- Peak flow chart - variability

- Lung function testing in the laboratory (including challenge test)

3. How do lifestyle factors such as smoking influence your decision?

Prompt list in case they are not raised:

- Smoking status

- Exercise

- Weight

4. Often, patient characteristics such as gender or age influence decisions we make. Are there patient characteristics that influence your decision to make a diagnosis of asthma?

Prompt list in case they are not raised:

- Age

- Gender

- Cough after infection - Post viral? Asthma? Something else?

- History of atopy

- Occupation

- Pets

- Anxiety

- Ethnic background

- Socioeconomic status

- Childhood asthma / childhood respiratory problems

- They way in which they describe their signs or symptoms.

- Co-morbidity.

5. How might the ethnic background of the patient affect your diagnosis? If a patient is from an ethnic background that has a low prevalence of asthma and presents with respiratory symptoms, how might this affect how you think?

Reflect on issues raised

6. Are there any issues we have not touched on yet that might contribute to difficulties or ease of diagnosing asthma? 